\title{
Effect of Two Natural Antioxidant Intervention on Plasma Vitamin C, E, and $\beta$-Carotene Concentrations of Apparently Healthy Adults in Nasarawa State University, Keffi, Nigeria
}

\author{
Chibuzo Carole Nweze ${ }^{1, *}$ and Miriam Solomon ${ }^{2}$
}

\author{
${ }^{1}$ Department of Biochemistry and Molecular Biology, Nasarawa State University, Keffi, Nigeria \\ ${ }^{2}$ Department of Biochemistry, College of Medical Sciences, University Jos Nigeria
}

Postal address: Department of Biochemistry and Molecular Biology, Faculty of Natural and Applied sciences, Nasarawa State University, Keffi, P.M.B. 1022 Keffi, 93001, Keffi, Nigeria.

Phone no: 2348036091147

Email address: chibuzoihe@gmail.com; danladis2003@yahoo.com

Keywords: Blood, antioxidants, aging, gender, micronutrients

\begin{abstract}
Immunologic functions particularly cell-mediated immunity declines with age, contributing to the increased incidence of infectious diseases in the elderly. The study looked at vitamin $C$, vitamin $E$, and $\beta$-carotene levels in plasma of 150 healthy adults of 96 men and 54 women aged between 30 and 74 years. The subjects were randomly grouped into three at baseline for further dietary studies, The antioxidant dietary supplements were either 1 capsule per day of antioxidant nutraceutical (Forever living product) (containing vitamin E $10 \mathrm{mg}$, vitamin C 60mg and $\beta$ - carotene $2000 \mathrm{mcg}$ of vitamin A, or cheap affordable indigenous antioxidant functional foods of equivalent vitamin composition of oranges, carrots, and soybean or bottled water, and the first dose was dispensed and followed up for six months. The result showed that the vit $\mathrm{C}, \mathrm{E}$ and $\beta$ carotene concentration of antioxidant treated groups were positively influenced than the placebo group (Grp 1 (AT-P) 6m. Grp 3 (AT-F) 6m was affected more positively than the antioxidant Grp 2 $(\mathrm{AT}-\mathrm{N})_{6 \mathrm{~m}}$. Vitamin $\mathrm{C}, \mathrm{E}$, and $\beta$-carotene concentrations of the elderly are beyond reference/normal range of blood plasma. Plasma Vitamin $C$ and $\beta$-carotene concentration of the female was higher than the male. The vitamin E concentration in blood plasma of male is higher compare to female.
\end{abstract}

\subsection{INTRODUCTION}

Immunologic functions particularly cell-mediated immunity declines with age, contributing to the increased incidence of infectious diseases in the elderly. Cellular immune functions and health generally are compromised at older age due to severe nutritional deficiency. Nutrition may play a pivotal role in maintaining immune competence in older adults. The maintenance of immune cells and preserving their adequate function are strongly influenced by antioxidant/oxidant balance. Thus, the level of anti-oxidants in these cells plays a pivotal role in protecting them from oxidative stress [1].

Free radicals are incriminated in the pathogenesis of tissue injury in many diseases in ageing, as well as obstruction of normal physiological functions. They produce cellular injury by lipid peroxidation, enzyme activation, DNA damage and degradation of structural protein. The body has evolved multiple defence mechanism through antioxidants against free radicals, by preventing the formation of radicals (chain termination), scavenging them or promoting their decomposition [2]. This enzymatic and non-enzymatic antioxidant includes Vitamin C, E, A, carotenoids, superoxide dismutase (SOD), catalase, glutathione peroxidase (GPx). New concept of the application/approach of antioxidant supplement has appeared as nutraceuticals, functional foods, nutritional therapy, and phytonutrients [3] [4]. Whole foods represent the simplest example of functional foods (carrots, orange, soybeans and avocado pear) because of their high contents of physiologically active components ( $\beta$ - carotene, Vitamin $\mathrm{C}$, Vitamin E, and Lycopene respectively). They satisfactorily demonstrate a beneficial effect on one or more target functions in the body, beyond adequate nutritional effect, relevant to well-being and reduction to health risk [5]. More specifically, 
antioxidants maintain the integrity and function of membrane lipids, cellular proteins, and nucleic acids and the control of signal transduction of gene expression in immune cells. For this reason the immune cells are particularly sensitive to changes in their antioxidant status.

Antioxidant vitamins (Ascorbic acid and tocopherol) and carotenoids have been suggested to play important role in cellular immunity and cardiovascular diseases [6] [7] [8]. Vitamin $\mathrm{C}$ is found in high concentration in white blood cells and is rapidly utilized during infection; reduced plasma concentrations are often associated with reduced immune function [9] Vitamin C provide important protection for plasma lipid and lipid membranes and can neutralize phagocyte-derived oxidants released extracellular, thereby preventing oxidant mediated tissue damage [10]. Vitamin $\mathrm{E}$ is the most effective chain-breaking, lipid soluble antioxidant present in cell membranes, thus play a role in limiting lipid peroxidation as well as in immune cell function [11]. $\beta$-carotene in a study was shown to have significant antioxidant properties and effectively quenches singlet oxygen free radicals than vitamin A [12]. B-carotene and some other antioxidant carotenoids can enhance many aspects of immune functions [13]. Micronutrients deficiency is common in ageing, pregnancy and ill health [14]. These deficiencies may contribute to an increased risk of parasitic infection, and oxidative stress which affect the production of blood cells and haemoglobin and the entire immune level [15]. Biochemical investigation of average Nigerians of different ages and of both sex will be carried out, with regards to serum level of specific micronutrients. on cellular immunity. This study is focused on the evaluation of blood biochemical analysis of humans on micronutrients (Vitamin $\mathrm{C}, \mathrm{E}$, and $\beta$ - carotene) of adults on different ages.

\subsection{MATERIALS AND METHODS}

The study was conducted in Keffi town, Nasarawa State, Nigeria. A single- centered open dose study was employed. The study subjects were a total of 150 healthy adults of 96 men and 54 women aged between 30 and 74 years, all volunteers are staff (academic staff, senior staff, and nonacademic) of Nasarawa state University, Keffi. The subjects had no history of gastrointestinal surgery, or other significant pathology, were not on a caloric- reduced or vegetable diet nor were taking any antioxidant/vitamin supplement, female were not pregnant or lactating. No concomitant medication was allowed throughout the study except the contraceptive pill. The protocol was reviewed and approved. The scope, nature, aim and objectives of this study were thoroughly explained to voluntary participants for their consent, and were all made to sign an informed consent letter and a questionnaire.

Three hundred (300) volunteers' veinous blood samples were taken by local physicians from the university staff clinic and clinical records were taken. Participants with desirable health status were chosen randomly. The volunteers were randomly assigned to three groups with 3 sub-groups based on two intervals of baseline and six months. Group 1; No antioxidant treatment was given for the six months but a $75 \mathrm{cl}$ bottled water- placebo group ( Grp $1(\mathrm{BT})_{0 \mathrm{~m}}$ : placebo basaline group, Grp 1 $(\text { AT-P })_{6 \mathrm{~m}}$ : placebo six months group.. Group 2; the group that later received antioxidant nutraceuticals Forever living product 1 capsule per day of (vitamin E (10mg), vitamin C (60mg) and $\beta$ - carotene $\left(2,000 \mathrm{mcg}\right.$ of vit A)-nutraceutical treatment group (Grp $2(\mathrm{AT}-\mathrm{N})_{0 \mathrm{~m}}$ : baseline group that were later given antioxidant nutraceuticals, Grp 2 (AT.N) $6 \mathrm{~m}$ : nutraceutical treatment group that received antioxidant for six months. Group 3; the group that later received antioxidant functional foods of equivalent vitamin composition oranges $(100 \mathrm{~g})$, carrots $(100 \mathrm{~g}), 75 \mathrm{cl}$ soybean drink and a heaped tablespoon of powdered soybean $(35 \mathrm{~g})$ daily-functional group treatment group (Grp 3 (AT$\mathrm{F})_{0 \mathrm{M}}$ : baseline group that was later given antioxidant, Grp $3(\mathrm{AT}-\mathrm{F})_{6 \mathrm{M}}$ : functional food treatment group that received antioxidant for six months.

Blood biochemical analysis/assays on micronutrients and $\beta$-carotene assay were carried out at Nigerian Institute of Pharmaceutical Research and Development, Idu Industrial District, Abuja, Nigeria. Determination of Micronutrients ( $\beta$-carotene, Vitamin E and Vitamin C in blood plasma) were measured by the method of Simultaneous determination of vitamins $C, E$ and $\beta$-carotene in human plasma by high performance liquid chromatography with photodiodes-array detection 
[16].Based on distribution equilibrium which is the differences in solubility and adsorption of a component in two immiscible phases, based on separation science in which a liquid mobile phase is percolated through a column.

\section{Statistical analysis}

The experimental results were expressed as the mean \pm SD. The parameters were analysed statistically using one-way analysis of variance (ANOVA) for comparing the mean using Smith's Statistical package (SSP).

\subsection{RESULTS AND DISCUSSION}

Table 1showed that the vit c concentration of antioxidant treated groups was positively influenced than the placebo group (Grp 1 (AT-P) $6 \mathrm{~m}$. Grp 3 (AT-F) $6 \mathrm{~m}$ were affected more positively than the antioxidant Grp $2($ AT-N) $6 \mathrm{~m}$. The results showed that vit c concentration of most of the elderly were lower than that of the younger adults. Also, vit c concentration of the female was higher than that of male. The vit c concentration of most on the age range 60-79 are beyond reference/normal range of blood plasma vit $\mathrm{c}$ in both male and female but increased positively to the reference range after the antioxidant intervention. The serum vitamin C of Grp 1 (AT-P) at six months showed a significant different at the age groups except for the both sexes of age group 30-39, male of age group 40-49 and female of age group 60-69 while at twelve months there was significant difference in all it was noted that at extreme ages there was negative increament. Grp 2 (AT-N) at six showed a significant difference in all ages of males and females. Grp 3 (AT-F) at six months also showed a significant difference in all ages of males and females.

The vit E concentration of the antioxidant treated groups was positively influenced than the placebo group (Grp 1 (AT-P) at six months. Grp 3 (AT-F) at six months was affected more positively than the antioxidant Grp 2 (AT-N) at six months. The result showed that the males have higher levels of vit $\mathrm{E}$ compare to females of the same age. It was noted that the vit E concentration in blood plasma decreases with increase in age. The serum vitamin E of Grp 1 (AT-P) ${ }_{6 m}$ showed a significant different in negative decrease at the age groups when compared to Grp 1 (BT) except for the male age group of 30-39, 40-49, 50-59 and 60-69. while Grp 2 (AT-N) at six months showed a significant difference in positive increase. Grp 3 (AT-F) at six months showed a significant difference at all the age groups of males and females.

The $\beta$-carotene concentration of antioxidant treated groups at six months was positively influenced than the placebo group (Grp 1 (AT-P) at six months. Grp 3 (AT-F) at six months was affected more positively than the antioxidant Grp $2(\mathrm{AT}-\mathrm{N})$ at six months. The result showed that the females have higher and normal levels of $\beta$-carotene compare to males of the same age. The result showed that most elderly (50-79) has low $\beta$-carotene concentration lower than the normal range in blood plasma and that it decreases with increase in age. The serum $\beta$-carotene of Grp 1 (AT-P) $6 \mathrm{~m}$ showed a significant difference in negative decrease at all the age groups except for both sexes of age group 40-49, male of age group 50-59 and female of age group 60-69. Grp 3 (AT-F) at six months showed a significant different in positive increase at all age groups of males and females. Grp 2 (AT-N) at six months showed a significant different in positive increase at all age groups of male and females. This study showed that the antioxidant dietary intervention significantly improved these micronutrients and $\beta$-carotene levels in blood, with antioxidant functional foods affecting the micronutrients and $\beta$-carotene better than antioxidant nutraceuticals. Micronutrients deficiency is common in ageing, pregnancy and ill health [14]. These deficiencies may contribute to an increased risk of parasitic infection, and oxidative stress which affect the production of blood cells and haemoglobin and the entire immune level [10]. The study showed that there is synergism between $\alpha$-tocopherol, $\beta$-carotene and ascorbic acid. Ascorbic acid acts in an aqueous phase [14], whereas $\alpha$ tocopherol and $\beta$-carotene act in the lipophilic compartments [13]. Chemically, $\beta$-carotene is less reactive toward radicals than are $\alpha$-tocopherols and ascorbic acid [12]. Ascorbic acid and $\alpha$ tocopherol inhibit oxidation synergistically [11]. Though $\alpha$ - tocopherol is more effective than ascorbic acid in scavenging radicals in membrane and lipoproteins [12]. Ascorbic acid reduces the 
resulting $\alpha$-tocopheroxyl radicals [12]. $\alpha$-tocopherol and $\beta$-carotene may be assumed to have a cooperative effect by residing and scavenging radicals at different positions in the compartment [13]. In conclusion, the combination of $\alpha$-tocopherol, $\beta$-carotene and ascorbic acid can be said to be effective in inhibiting oxidative damage which showed in the cellular immunity of the volunteers in the antioxidant intervention groups studied.

Vitamin $\mathrm{C}$ concentration in plasma showed no significant difference in ages of male compared to female (Table 1), indicating that female has higher Vitamin C concentration. Also, the vitamin C concentration of the elderly was beyond the normal range, showing lower vitamin than that of the adults. Some studies indicate that Vitamin $\mathrm{C}$ increases circulating immunoglobulin (Ig) levels in humans [17]. Vitamin C provide important protection for plasma lipid and lipid membranes and can neutralize phagocyte-derived oxidants released extracellular, thereby preventing oxidant mediated tissue damage [10]. This study showed that Vitamin $\mathrm{C}$ concentration showed no significant difference between the male and female levels. . This study conform to previous study in Helena that Vitamin $\mathrm{C}$ evaluation on blood of volunteers for cancer research that the female has higher Vitamin C level than male [18] [19]. Vitamin C level of elderly has shown to be lower than that of the young adults.

Table 2 showed that Vitamin E level significantly decreases with increase in age of both male and female. A study showed that there was a negative association between plasma Vitamin E levels and incidence of infections in healthy adults aged over 60years [20]. The latter effect is probably due to the increase in production of free radicals and related species in ageing [21], and tends to increase from infancy to adolescent [20]. Result shown in Table 3 obtained on $\beta$-carotene concentration showed that the female $\beta$-carotene is significantly higher than in male. The result also showed that $\beta$ - Carotene decreases with age. In respect to age, the study conforms to report on micronutrients of adults that $\beta$-carotene concentration of male and female decrease with age [22].

Aging which is a multifactorial process in which free radical oxidative damage plays a very important role and antioxidant defence mechanisms in humans, such as antioxidative enzymes, tocopherol, ascorbic acid and $\beta$-carotene are linked to each other balance with reactive oxygen species[22]. The study showed that all parameters evaluated and assayed increase/decrease with increased in age to the extreme value of the normal/reference range. Generally, the abnormal increase and decrease of these various biochemical parameters in the blood can be improved by increasing concentration of dietary foods containing antioxidant. This deficiency greatly affects the immune system and increases the rate of ageing of individuals of either sex.

\section{ACKNOWLEDGEMENT AND FUNDING}

The volunteers (staff of Nasarawa State University, Keffi),staff of Innovative Biotechnology and Research Laboratory, Keffi, staff at the Medicinal Chemistry and Quality Control department of Nigerian Institute of Pharmaceutical Research and Development, Idu Industrial Layout, Abuja are all appreciated.

This study was supported by grants from Tertiary Education Trust Fund, Nigeria. The funders had no role in study design, data collection and analysis, decision to publish, or preparation of manuscript for publication of this work.

\section{CONFLICT OF INTEREST}

The authors declare no conflict of interest. 
Table 1: Effect of antioxidants on Serum Vitamin C ( $\mu \mathrm{mol} / \mathrm{L})$

\begin{tabular}{|c|c|c|c|c|c|c|c|c|c|c|c|c|c|}
\hline & GROUP & Grp1(B & $0 \mathrm{~m}$ & Grp2( & $0 m$ & Grp3 (I & T)0m & Grp1(A & Г-P)6m & Grp2(A & $\Gamma-N) 6 m$ & Grp2(A & [-F)6m \\
\hline & SEX & $\mathrm{M}$ & $\mathrm{F}$ & $\mathrm{M}$ & $\mathrm{F}$ & $\bar{M}$ & $\bar{F}$ & $\overline{\mathrm{M}}$ & $\bar{F}$ & $\overline{\mathrm{M}}$ & $\bar{F}$ & $\overline{\mathrm{M}}$ & $\bar{F}$ \\
\hline \multirow{10}{*}{ AGES } & \multirow[t]{2}{*}{$30-39$} & 42.00 & $46.00 \pm$ & 40.00 & $40.00 \pm$ & 41.00 & 46.00 & $42.00 \pm$ & 46.00 & $46.00 \pm$ & $57.0 \pm$ & $48.0 \pm$ & $58.00 \pm$ \\
\hline & & \pm 0.03 & 0.03 & \pm 0.02 & 0.02 & \pm 0.03 & \pm 0.02 & 0.03 & \pm 0.03 & $0.02^{\mathrm{b}}$ & $0.04^{\mathrm{b}}$ & $0.04^{\mathrm{c}^{*}}$ & 0.03 \\
\hline & \multirow[t]{2}{*}{$40-49$} & 40.00 & $45.00 \pm$ & 39.00 & $45.00 \pm$ & $40.00 \pm$ & 45.00 & $40.00 \pm$ & $46.0 \pm$ & $45.00 \pm$ & $55.0 \pm$ & $47.0 \pm$ & $57.0 \pm$ \\
\hline & & \pm 0.05 & 0.09 & \pm 0.07 & 0.10 & 0.06 & \pm 0.09 & 0.06 & $0.07^{\mathrm{a}}$ & $0.06^{\mathrm{b}}$ & $0.05^{\mathrm{b}}$ & $0.05^{\mathrm{c}^{*}}$ & $0.05^{\mathrm{c}}$ \\
\hline & \multirow[t]{2}{*}{$50-59$} & 41.00 & $44.00 \pm$ & 40.00 & $45.00 \pm$ & $40.00 \pm$ & 44.00 & $42.00 \pm$ & $45.0 \pm$ & $45.00 \pm$ & $55.0 \pm$ & $46.00 \pm$ & $56.0 \pm$ \\
\hline & & \pm 0.10 & 0.10 & \pm 0.09 & 0.08 & 0.09 & \pm 0.10 & $0.03^{\mathrm{a}}$ & $0.05^{\mathrm{a}}$ & $0.05^{\mathrm{b}}$ & $0.06^{\mathrm{b}}$ & $0.06^{\mathrm{c}}$ & $0.06^{\mathrm{c}}$ \\
\hline & \multirow[t]{2}{*}{$60-69$} & 40.00 & $45.00 \pm$ & 40.00 & $44.00 \pm$ & $41.00 \pm$ & 44.00 & $41.00 \pm$ & 45.00 & $41.00 \pm$ & 45.00 & $48.0 \pm$ & $56.0 \pm$ \\
\hline & & \pm 0.06 & 0.06 & \pm 0.05 & 0.05 & 0.05 & \pm 0.06 & $0.02^{\mathrm{a}}$ & \pm 0.02 & $0.02^{\mathrm{a}}$ & \pm 0.02 & $0.03^{\mathrm{c}^{*}}$ & $0.03^{\mathrm{c}^{*}}$ \\
\hline & \multirow[t]{2}{*}{$70-79$} & 33.00 & $42.00 \pm$ & 29.00 & $43.00 \pm$ & $31.00 \pm$ & 43.00 & $31.00 \pm$ & $40.0 \pm$ & $36.00 \pm$ & $53.0 \pm$ & $38.00 \pm$ & $55.00 \pm$ \\
\hline & & \pm 0.03 & 0.00 & \pm 0.00 & 0.03 & 0.04 & \pm 0.00 & $0.01^{\mathrm{a}}$ & $0.00^{\mathrm{a}}$ & $0.00^{\mathrm{b}}$ & $0.01^{\mathrm{b}}$ & $0.01^{\mathrm{c}^{*}}$ & $0.00^{\mathrm{c}^{*}}$ \\
\hline
\end{tabular}

Reference value: 36.1-79.4 ( $\mu \mathrm{mol} / \mathrm{L})$; Grp 1 (BT): Group 1 before treatment; Grp 2 (BT): Group two before treatment; Grp 3 (BT): Group 3 before treatment; Grp 1 (AT-P) 6m: Group 1 after treatment- placebo; Grp 2 (AT-N) 6m: Group 2 after treatment- nutraceuticals; Grp 3 (AT-F) 6m: Group 3 after treatment- functional food; $\mathrm{p}<0.05$ was considered statistically significant; $\mathrm{n}: 150$; ${ }^{a}$ : was considered statistically significant when compared with Grp 1 (BT); ${ }^{\text {b: }}$ was considered statistically significant when compared with Grp $2(\mathrm{BT}){ }^{\mathrm{c}}$ : was considered statistically significant when compared with Grp 3 (BT); ${ }^{*}$ : was considered statistically significant when compared with Grp 2 (AT-F) $6 \mathrm{~m}$

Table 2: Effect of antioxidants on Serum Vitamin E ( $\mu \mathrm{mol} / \mathrm{L})$

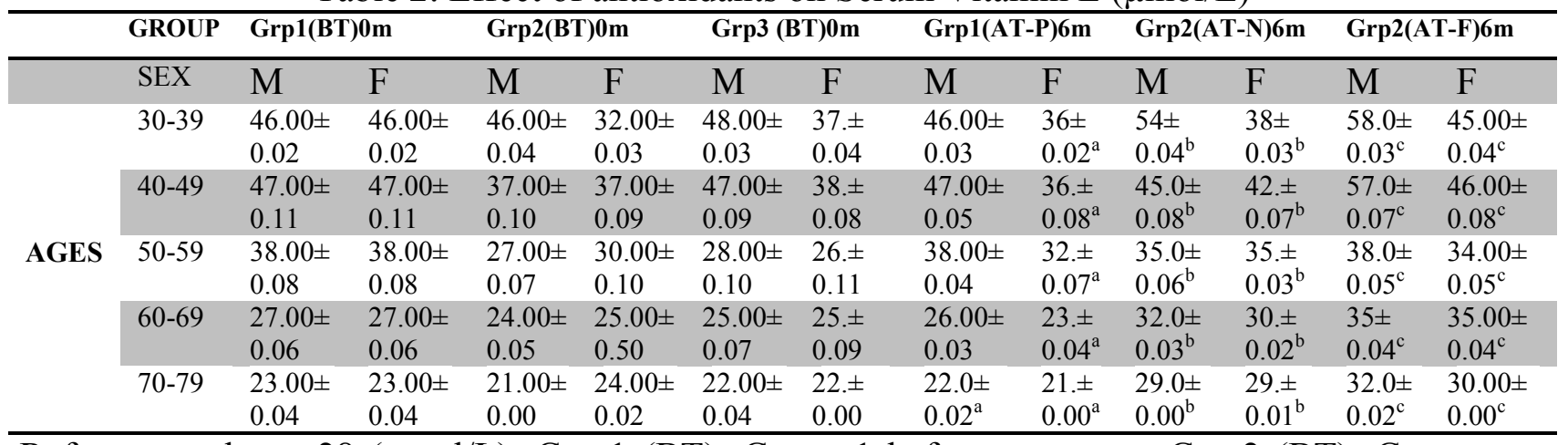

Reference value: $>28(\mu \mathrm{mol} / \mathrm{L})$; Grp 1 (BT): Group 1 before treatment; Grp $2(\mathrm{BT})$ : Group two before treatment; Grp 3 (BT): Group 3 before treatment; Grp 1 (AT-P) ${ }_{6 m}$ : Group 1 after treatmentplacebo; Grp 2 (AT-N) 6m: Group 2 after treatment- nutraceuticals; Grp 3 (AT-F) 6m $_{6}$ : Group 3 after treatment- functional food; $\mathrm{p}<0.05$ was considered statistically significant; $\mathrm{n}: 150$; ${ }^{\text {a }}$ : was considered statistically significant when compared with Grp $1(\mathrm{BT}){ }^{\text {b }}$ : was considered statistically significant when compared with Grp 2 (BT); ${ }^{c}$ : was considered statistically significant when compared with Grp 3 (BT); ; : was considered statistically significant when compared with Grp 2 (AT-F) $6 \mathrm{~m}$.

Table 3: Effect of antioxidants on Serum $\beta$ - Carotene ( $\mu \mathrm{mol} / \mathrm{L})$

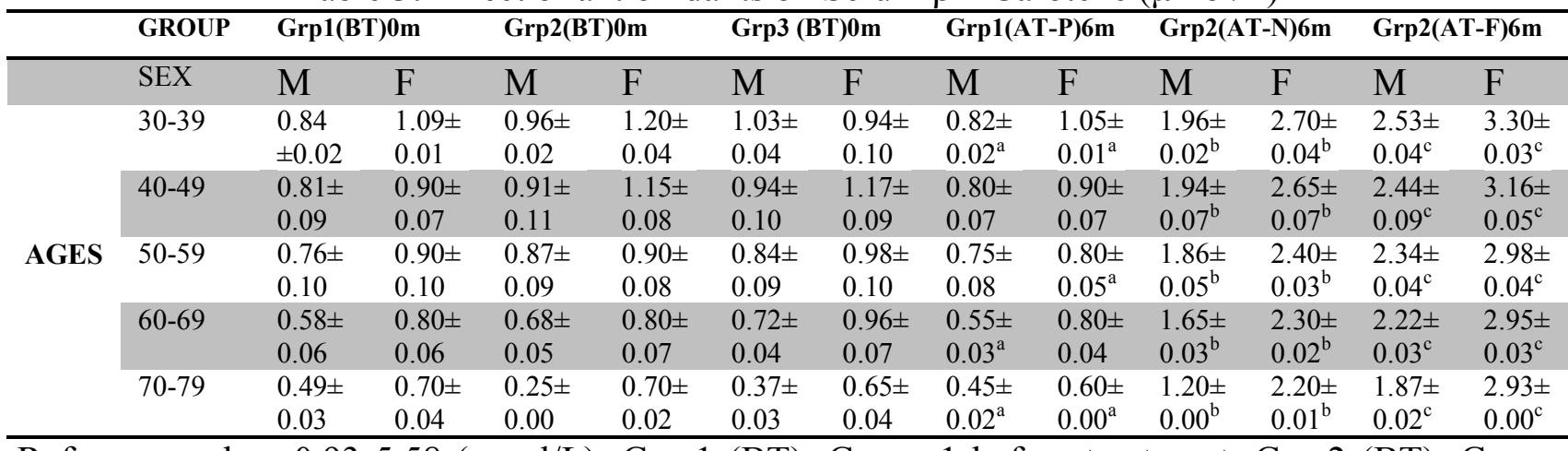

Reference value: 0.93-5.58 ( $\mu \mathrm{mol} / \mathrm{L})$; Grp 1 (BT): Group 1 before treatment; Grp 2 (BT): Group two before treatment; Grp 3 (BT): Group 3 before treatment; Grp 1 (AT-P) 6m: Group 1 after treatment- placebo; Grp 2 (AT-N) 6m: Group 2 after treatment- nutraceuticals; Grp 3 (AT-F) 6m: Group 3 after treatment- functional food; $\mathrm{p}<0.05$ was considered statistically significant; $\mathrm{n}$ : 150; 
a: was considered statistically significant when compared with Grp 1 (BT); ${ }^{\text {b: }}$ was considered statistically significant when compared with Grp 2 (BT); ${ }^{c}$ : was considered statistically significant when compared with Grp 3 (BT); ${ }^{*}$ : was considered statistically significant when compared with Grp 2 (AT-F) 6 m.

\section{REFERENCES}

[1] Knight, J., Free radicals their history and current status in aging and disease. Ann Clin Lab Sci., 28: pp.331-346 (1998).

[2] Shi, H.L., et al., Comparative study on dynamics of antioxidative action of $\alpha$-tocopheryl hydroquinone, ubiquinol and $\alpha$-tocopherol, against lipid peroxidation. Free Radic Biol Med, 27: pp. 334-346 (1999).

[3] Bland, J.S., Phytonutrition, pyhtotherapy and phytopharmacology. Altern Ther Health Med., 2: pp. 73-76 (1996).

[4] Berger, M.M., and A. Shenkin, Vitamins and trace elements: Practical aspects of supplementation. Journal of Nutrition. 22: pp. 952-955 (2006).

[5] Lopez-Varela, S., Gonzalez-Gross, M., and A. Marcos, Functional foods and the immune system: A review.European Journal of Clinical nutrition, 56: pp. 529-540 (2002)

[6] Diplock, A.T., Antioxidants and Free radical Scavangers In: Free radical damage and its control. Rice Evans, C.A. and R.H. Burdon (eds). Elsevier Science, UK. pp. 113-129 (1994).

[7] Mates, J.M., N. Perez-Gomez, and I.N. De Castro, Antioxidant enzymes and human diseases. Journal of Clinical Biochememistry, 32: pp. 595-603 (1999).

[8] Ballali, S., and F. Lanciai, Functional food and diabetes: a natural way in diabetes prevention. International Journal of Food Science and Nutrition, 63(1): pp. 51-61 (2013).

[9] Siegel, P.M. and J. Massague, Cytostate and apoptotic activities of TGF- beta in homeostatis and cancer. Pubmed Nat.Rev Cancer, 3: pp. 807-821 (2003)

[10] Anderson, J., C.L. Salzer, and J.P. Kumar, Regulation of the retinal determination gene dachshund in the embryonic head and developing eye of drosophilla. Journal of Developmental Biology, 297(2): pp. 536-549 (2006).

[11] Meydani, S.N., M. Meydani, and J.B. Blumberg, Vitamin E supplementation and in vivo immune response in healthy subjects: 8 randomisrd controlled trial. JAMA, 227: pp. 1380-1386 (1997).

[12] Saloneu, R.M., Six year effect of combined vitamin C, E, and $\beta$ - carotene supplementation on artheriosclerotic progression. Circulation, 1: pp. 947-953 (2003).

[13] Gardner, E.M., et al., The age-associated decline in immune function of healthy individuals is not related to changes in plasma concentrations of $\beta$-carotene, retinol, $\alpha$-tocopherol or zinc, Mechanism in Ageing. Journal of clinical Biochemistry, 94: 55-69 (1997).

[14] Stephen, L.S., M.C. Lathan, and E.A. Ottesen, Malnutrition and parasitic infections. International Journal of Parasitology, 121: pp. 532-538 (2000).

[15] Mahomed, K., Iron supplementation in pregnancy. Rev, 2: pp. 11-17 (2000).

[16] Zhao, B., et al., Simultaneous determination of vitamins C, E and b-carotene in human plasma by high performance liquid chromatography with photodiodes-array detection. Journal of pharmaceutical science, 30: pp. $72-87$ (2004).

[17] Stardam, E.R., Role of oxidant species in aging. Curr. Med. Chem., 11: pp. 1105-1112 (2004).

[18] Smirnoff, N., L-ascorbic acid biosynthesis. Vitam Horm, 61: 241-266 (2001). 
[19] Sies, H. and W. Stahl, Vitamins E, C, and $\beta$ - carotene, and other carotenoids as antioxidants. American Journal of clinical Nutrition, 62: pp. 1315s-1321s (1995).

[20] Soko,1 R.J., Vitamin E deficiency and neurologic diseases. Annu. Rev. Nutr., 8: pp. 351-373 (1988).

[21] Meydani, S.N., Barklung, M.P. and S. Siu, Vitamin E supplementation enhancers' cellmediated immunity in healthy elderly subjects. American Journal of. Clinical Nutrition, 52: pp. 557-563 (1990).

[22] Ventura, M.T., In vitro vitamin $\mathrm{E}$ and $\beta$-carotene, selenium supplementation improves neutrophil-mediated functions and monocyte chemoattractant proteins-1 production in the elderly. Cytobios, 77: pp. 225-232 (1994). 